\title{
FE BONE STRUCTURAL ANALYSIS WITH CT MAPPING OF INHOMOGENEOUS MATERIAL PROPERTIES
}

\author{
M. T. Bahia ${ }^{1}$, E. G. F. Mercuri ${ }^{2}$, and M. B. Hecke ${ }^{3}$ \\ ${ }^{1}$ Federal Institute of Santa Catarina \\ Rua Pavão, 1377, Bairro Costa e Silva - CEP 89220-618, Joinville, SC, Brasil \\ e-mail: migueltbahia@ifsc.edu.br \\ ${ }^{2,3}$ Federal University of Paraná \\ Centro Politécnico - Bloco Lame/Cesec, Caixa postal 19011, Bairro Jardim das Américas CEP 81531- \\ 990, Curitiba, PR, Brasil \\ \{emiliomercuri, mildredhecke\}@gmail.com
}

Keywords: Subject-specific Finite Element Modeling, Computed Tomography (CT), Image Processing, Bone Biomechanics, Young Modulus, Femur.

\begin{abstract}
This paper presents a bone structural analysis of a reconstructed fractured femur assigning inhomogeneous isotropic material properties accessed from CT images into the FE model. The grayscale values accessed from CT images were converted into local Young modulus that was assigned to the associated finite element of the mesh. The FE model was obtained from CT images by a numerical reconstruction of a proximal femur which presents an atypical fracture in the sub-trochanteric region with full displacement and no continuity between the fragments. The reconstruction involved procedures such as volume reconstruction from images, segmentation by thresholding, labeling of the connected components and also the alignment of point clouds data using the Iterative Closest Point (ICP) algorithm. The resulted aligned point cloud was thus converted into a solid and finally in a finite element mesh, that was analyzed in a standard FE framework. Another FE model with constant elastic modulus for cortical and trabecular bone was also simulated to compare results with our model. The values of principal stress and strains were rather closer to tensile values but for compressive values there were obtained significant differences between the two models, indicating that inhomogeneous model captures better the mechanical behavior for compression. Results obtained are in accordance with expected bone biomechanics behavior. The assignment of inhomogeneous material properties into the FE model become the structural analyzes more realistic and this research highlight that this assignment is fundamental for reliable subjectspecific models. The procedure implemented for reconstruction of fractured bones using CT images offers new insights to analyze the bone fractures since allows recovering the material state immediately before the failure.
\end{abstract}




\section{INTRODUCTION}

The modeling of biological tissues has commonly involved the adoption of homogeneous isotropic materials, with constant Young's modulus and Poisson's ratio. Such adoption has been very useful considering aspects such as the simplicity of computational implementation and the theoretical consolidation in the scientific community. However, in the case of bone, for instance, many studies highlight that this tissue presents heterogeneous physical properties and anisotropic behavior with age and tissue density dependence [1].

On the other hand, the use of subject-specific FE models as a tool to access the state of stress and fracture risk in individual patients based on CT images is increasing among researchers [2-4]. In this perspective, the accurate knowledge of the stress and strain characteristics implies the use of reliable models that consider specific features to define the geometric and the material model.

The method used to perform CT scans is accurate; the test is non-invasive, painless and involves little radiation exposure for patients allowing acquiring images with high quality from any part of human body, even in very small structures such as, for example, brain detail.

The CT numbers or the intensity of image pixels (16 bits) is represented by Hounsfield Units (HU). The HU numbers describe quantitatively a radiological density, which corresponds to a linear transformation of measuring the linear attenuation coefficient. The radiological density of distilled water at standart temperature and pressure is set to $0 \mathrm{HU}$ (zero HU) and the radiological density of air at STP is set to $-1000 \mathrm{HU}$. The HU numbers can be related to an apparent density using hydroxyapatite phantoms of known mineral density, and further performing a linear interpolation. There are several relationships between the HU values and Young modulus available at the literature, for instance in [5-6]. Thus it is possible perform a mapping into the inhomogeneus isotropic material properties and further assign the material properties into the FE model. In this work we have implemented a procedure to perform the mapping of the grayscale values accessed from CT images, conversion into bone ash densities and Young modulus and assignment of these local material properties into the FE model. Similar mapping of properties accessed from CT images have been performed by softwares like IA-FEmesh $^{1}$ and Bonemat ${ }^{2}$ [7]. However this kind of mapping is commonly used when there is geometrical equivalence and equal reference coordinates between the images and the 3D model reconstructed.

In this work we perform a numerical reconstruction of a fractured femur preserving the grayscale values accessed from CT images. These grayscale values are correlated to bone ash densities and converted into local elastic modulus. This inhomogeneous isotropic material distribution is finally assigned to the FE model, which contribute to become the model more realistic and reliable. Besides, this numerical implementation of reconstruction of fractured bones using CT images offers new insights to analyze the bone fractures since allows recovering the material state immediately before the failure. The steps for reconstruction of the femur involved techniques of image processing such as segmentation by thresholding, contour definition and labeling of connected objects. The alignment of point clouds was implemented using an Iterative Closest Point (ICP) algorithm, commonly used for registration on CAD systems [8].

\footnotetext{
${ }^{1}$ Available to download at https://www.ccad.uiowa.edu/MIMX/projects/IA-FEMesh

${ }^{2}$ Available to download at http://www.bonemat.org/
} 


\section{MATERIALS AND METHODS}

A fractured proximal femur was automatic 3-D reconstructed from CT images. The dataset of the fractured femur was taken in vivo, for diagnostic reasons. The patient was a 60 years old woman with a medical history of osteoporosis, as shown in Fig. 1. The CT scans were performed by the Center for Diagnostic Imaging at Hospital Santa Catarina (SP, Brazil). The images were kindly provided by professor Marcelo de Medeiros Pinheiro, Department of Medicine of the Federal University of São Paulo (UNIFESP). For this reconstruction we use $243 \mathrm{CT}$ slices of the fractured proximal femur (diaphyseal femoral head) spaced at $1.5 \mathrm{~mm}$, which means likely $3 / 4$ of the femur. The fracture was concerned about cross producing lateral displacement and angularity between the two resulting fragments. The 3-D reconstruction, mapping and assignment of inhomogeneous isotropic material properties, generation of FE model and structural analysis were fully implemented with in-house programs written in $\mathrm{MatLab}^{3}$. The creation of the STL file used as an input data for mesh creation was developed using the platform MeshLab ${ }^{4}$ and the post-processing visualization were performed in the open source Paraview 5. 5 . In the following, the procedure employed is detailed and the methods for creating a FE model with inhomogeneous isotropic material properties are summarized.

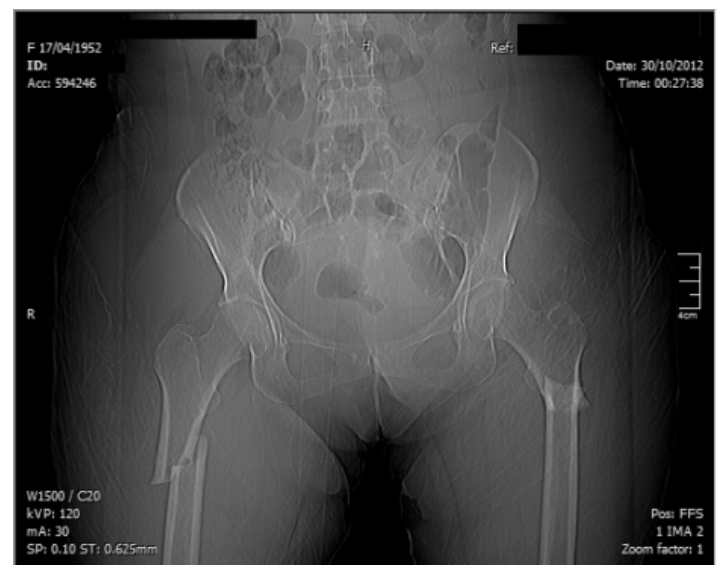

Fig. 1. CT scan of the atypical bilateral hip fracture without trauma. The donor is a female diagnosed with osteoporosis.

\subsection{Subject-specific FE model}

The CT scans were performed using a scanner with parameters: $120 \mathrm{kVp}, 30 \mathrm{~mA}, 1.5 \mathrm{~mm}$ slice thickness and pixel size of $0.32 \mathrm{~mm}$. In order to convert the image dataset to a 3D dataset of pixels, each image (that contains pixels information) is considered as a plane and each pixel a point in this plane. Acquiring planes at different depths so as the process is repeated, a $3 \mathrm{D}$ volume is acquired. Each level corresponds to the distance between each slice, as illustrated on Fig. 2.

After obtained the 3D coordinates others steps must be performed to isolate the part of 3D dataset to be analyzed. Here it was employed a segmentation by thresholding, which allows to remove all the pixels of a volume that do not satisfy a condition. In this case it was chosen an intensity threshold and only those pixels above the threshold were selected. The threshold

\footnotetext{
${ }^{3}$ Trademark of The MathWorks Inc., MA, USA.

${ }_{5}^{4}$ Available to download at http://meshlab.sourceforge.net/

${ }^{5}$ Available to download at http://www.paraview.org/
} 
used in this work was 104 for a grayscale range of $[0,255]$. Result of the segmentation can be viewed in Figs. 3 and 4.

Considering that in this case we are dealing with a fractured femur, it is necessary identify each fragment of the fractured femur and perform coordinates transformations in such a way that align the fragments to reconstruct the original femur before fracture. Translation of point cloud was carried out by adding the vector position of each node $i(i=1, \ldots$, number of nodes), the desired translation vector $\vec{T}=(a, b, c)$, where $a, b$, and $c$ are the displacements in $x, y$, and $z$, respectively.

$$
\vec{x}_{\text {new }}=\vec{x}_{\text {old }}+\vec{T}
$$

The rotation of the point cloud data about $x, y$, and $z$ axes is performed by multiplying the position vector of each node by the rotation matrix, wherein:

$$
\begin{array}{r}
R_{x}(\alpha)=\left[\begin{array}{ccc}
1 & 0 & 0 \\
0 & \cos \alpha & -\sin \alpha \\
0 & \sin \alpha & \cos \alpha
\end{array}\right] \\
R_{y}(\beta)=\left[\begin{array}{ccc}
\cos \beta & 0 & \sin \beta \\
0 & 1 & 0 \\
-\sin \beta & 0 & \cos \beta
\end{array}\right] \\
R_{z}(\gamma)=\left[\begin{array}{ccc}
\cos \gamma & -\sin \gamma & 0 \\
\sin \gamma & \cos \gamma & 0 \\
0 & 0 & 1
\end{array}\right]
\end{array}
$$

Unfortunately, the rotation matrices and translations vectors necessary to this alignment are unknown. To overcome this difficulty we employ here a registration technique for alignment of point cloud data called Iterative Closest Point method (ICP) that is commonly used in CAD systems for registration of surfaces. The ICP algorithm was introduced in 1991 by [9] and independently by [10] and it was further developed by various researchers.

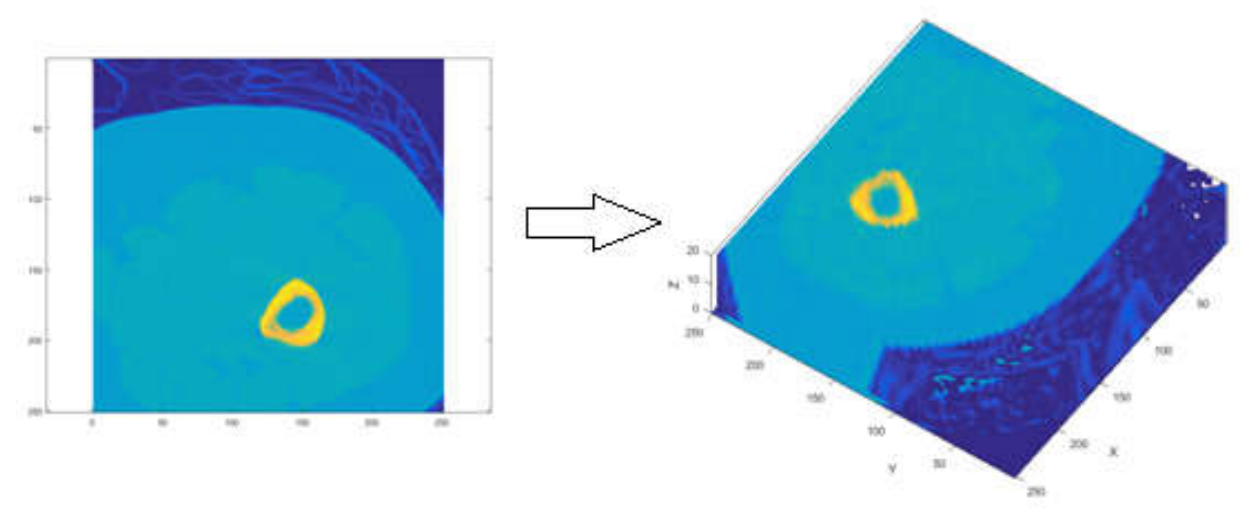

Fig. 2. Conversion of 2D CT images into a 3D volume.

The overall goal is to apply a transformation to one of the clouds and bring it as close to the other as possible. The convention is to apply the transformation to the data points in order to bring these to the best alignment with the model points. In 3-D space, such a transformation has six degrees of freedom - three rotations and three translations - and the objective function 
becomes a function of six variables, see [8], [11]. Expressing a rotation in terms of the rotation matrix $\mathbf{R}$ and a translation in the vector $\vec{T}$ the problem may be seen as a minimization problem of the squared distances from points in one cloud to their nearest neighbors in the other after appliance of the transformation. We are interested in to find $\mathbf{R}$ and $\vec{T}$ that minimize the following objective function:

$$
F=\sum_{i=1}^{N}\left\|[\mathrm{R}] p_{i}-\vec{T}-q_{i}\right\|^{2}
$$

In this work it was used as a reference point cloud a femur with no fracture. Thus, the fragments represented by point clouds were aligned using a healthy femur, to find the rotation matrix $\mathbf{R}$ and the translation vector $\vec{T}$. The resulted $3 \mathrm{D}$ aligned point cloud was converted into a 3D surface using the open source software Meshlab. The 3D surface was transformed in a solid finite element model discretized into 3846 nodes and 16689 linear tetrahedral elements, as illustrated on Fig. 6 .

\subsection{Mapping of pixel values in elements}

After performing the above procedures to reconstruct the fractured femur preserving the pixels information and generate the FE model, the next step is assign to each finite element of the mesh the average grayscale value of the pixels that fall inside of each element. Thus it is necessary to establish a criterion for checking if the pixel fall inside or outside of the tetrahedral finite element, and further assign this value (or average of values) locally to each element.
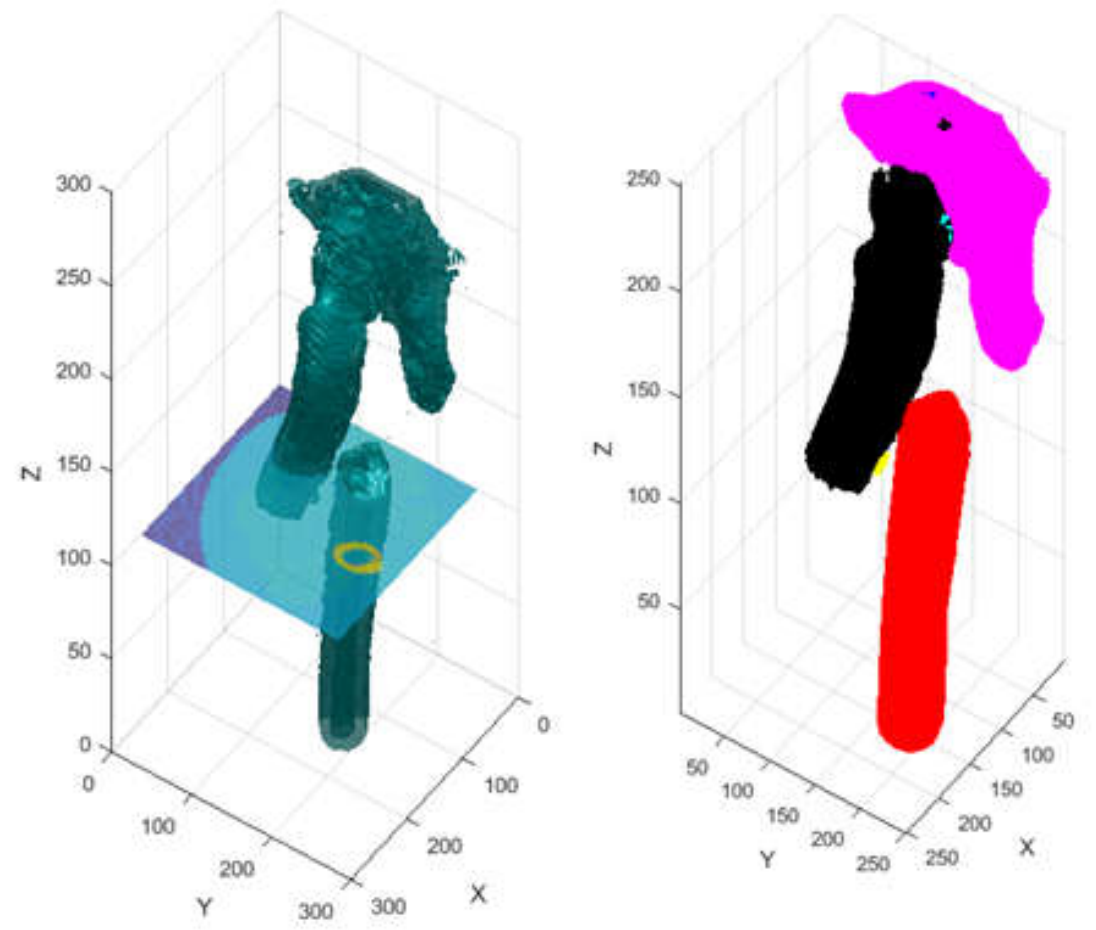

Fig. 3. Segmentation by thresholding (left) and labeling of connected components (right).

In other words, the goal here is to check whether a point defined by the pixels coordinates falls inside or outside of the convex hull defined by the vertices of each tetrahedral finite ele- 
ment and assigns the average of the grayscale values of the pixels inside the convex hull to each respective element. There are several possibilities to perform this. One very interesting way adopted here was proposed by [19] and is summarized as follows.

One point of a 3D point cloud data belongs to a specified tetrahedral if the signed volume of all the tetrahedral formed by this point and each triangular face of the specified element is positive. When at least one of the volumes of the tetrahedral formed by this point and each triangular face of the element is negative the point fall outside of the element. This procedure is repeated for all the elements of the model in order to verify if the point belongs or not to a specified element.

In this work we adopt medical images of 8 bits, representing a grayscale range of $[0,255]$. Fig. 5 shows a histogram of the distribution of the mapped pixels on the reconstructed femur by grayscale values provided by the $3 \mathrm{D}$ images. The bimodal appearance (two bells) of the graph indicates the two regions of bones: cortical and trabecular. Histograms such this one also allows to analyze the effects of CT image resolution in the material properties distribution. The result of the procedure of mapping and assignment of grayscale values into the FE mesh is illustrated at Fig. 6.
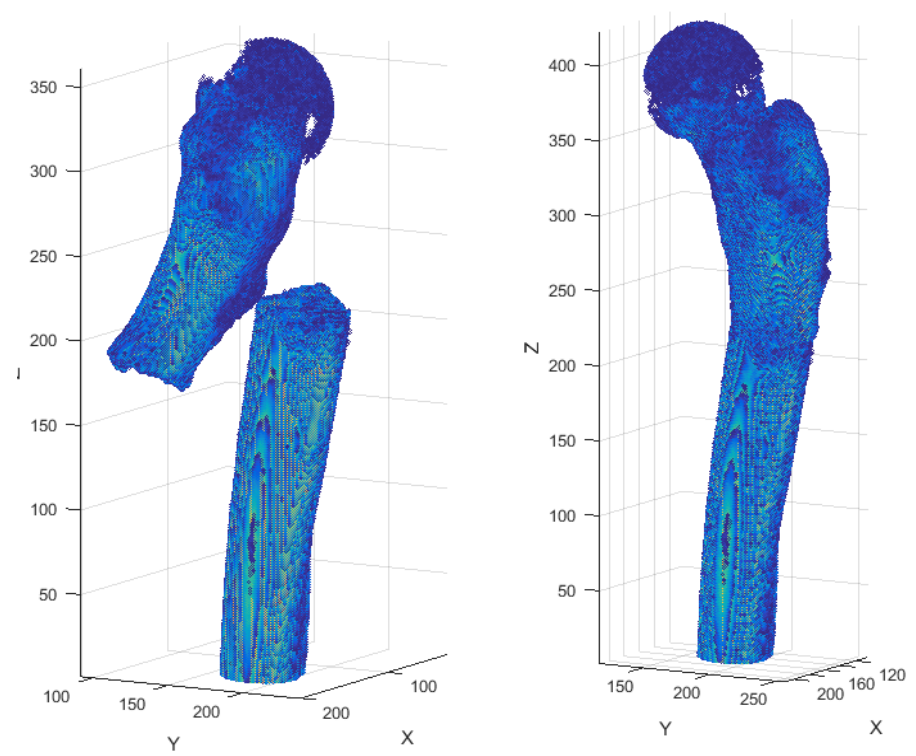

Fig. 4. Point cloud data of femora fragments (left) and aligned femora (right) using the ICP algorithm.

In order to determine the point wise Young modulus it was adopted the following procedure. For cortical bone, we assign to the whitest pixel of image (full bone with no voids) the value $1.4 \mathrm{~g} / \mathrm{cm}^{3}$ for bone ash density ${ }^{6}$. For water region on image (darkest pixel surrounding the bone) we attribute "zero" ash density. Other values of ash density were finding using a linear interpolation between the region with full bone with no voids $\left(\rho_{a s h}=1.4 \mathrm{~g} / \mathrm{cm}^{3}\right)$ and the water region ("zero" ash density), which produced the following equation:

$$
\left.\rho_{a s h}=p(x, y, z)-a\right] \quad \frac{14}{b-a}\left[\mathrm{~g} / \mathrm{cm}^{3}\right]
$$

where $p(x, y, z)$ is the image grayscale value at the position $(x, y, z), a$ and $b$ are the grayscale values for water and full bone with no voids, respectively. In this study, the values adopted were $a=66$ and $b=219$, considering a grayscale range of $[0,255]$. These values should be

\footnotetext{
${ }^{6}$ Ash weight divided by the real volume, which neglects the effect of fat content for trabecular bone.
} 
chosen according to the available images. After correlate the grayscale values with ash densities, it is adopted a relationship proposed by [16] and validated by [17] and [18] to determine the corresponding Young modulus (Eqs. 5 and 6):

$$
\begin{gathered}
E_{\text {cort }}=10200 \rho_{a s h}^{20}{ }^{1}[\mathrm{MPa}] \\
E_{\text {trab }}=5307 \rho_{a s h}+469[\mathrm{MPa}]
\end{gathered}
$$

For distinguish the cortical and trabecular bone, we define $\rho_{a s h}>06 \mathrm{~g} / \mathrm{cm}^{3}$ for cortical bone and $\rho_{a s h} \leq 06 \mathrm{~g} / \mathrm{c} \mathrm{m}^{3}$ for trabecular bone, as proposed by [12]. Poisson's ratio was set to 0.3 for all material bins, covering the range used in other studies, such as [12] and [15]. The elastic modulus range obtained with this procedure of mapping was approximately $1.06-$ $19.46 \mathrm{GPa}$.
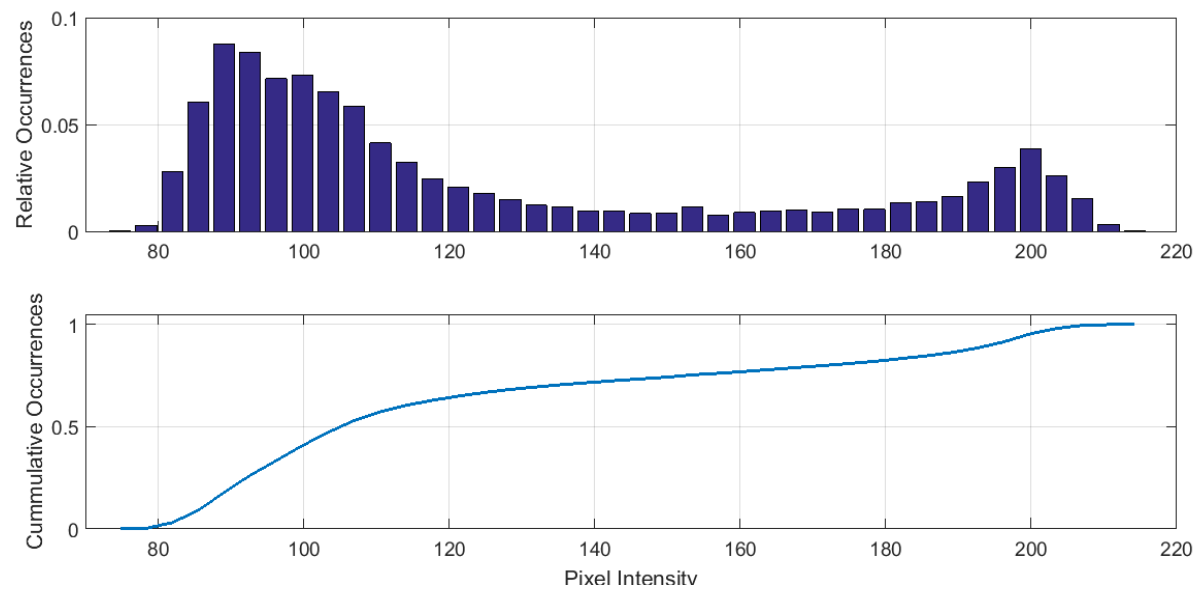

Fig. 5. Histograms of pixel intensities by relative and cumulative frequencies (above and behind, respectively).
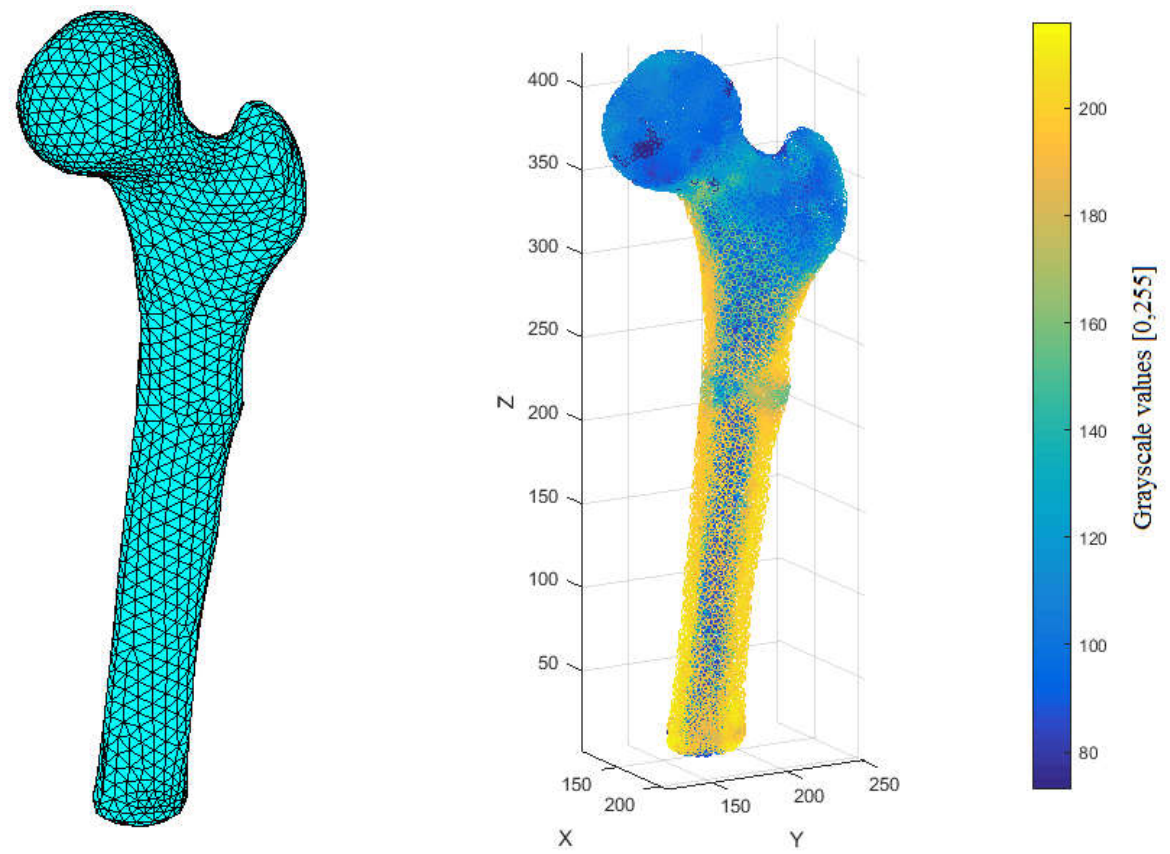

Fig. 6. Discretization of reconstructed femur in a tetrahedral finite elements mesh (left) and the assignment of the grayscale values for each finite element (right). 


\subsection{Boundary conditions of FE model}

Reliable boundary conditions are fundamental for a realistic analysis of a biomechanical model. Here the models proposed by [20] and [21], were modified to define the values of adductor force on the femur head and the abductor force on the greater trochanter. For this analysis we assume an individual weighting $680 \mathrm{~N}$. It is performed a balance of forces acting on the pelvis accordingly to [21], which establish the resultant adductor force upon femur head at an angle of $16^{\circ}$ with z-axis and a resultant abductor force upon greater trochanter at an angle of $22^{\circ}$ with z-axis. In this case, the adductor and abductor forces are $2168 \mathrm{~N}$ and $1632 \mathrm{~N}$, respectively. These forces were proportionally distributed on the element faces of the boundaries at femur head and greater trochanter as shown in Fig. 7. The value of each face force is proportional of the angle between the normal of the element face and the resultant force. The nodes on the basis of femur were set fixed.
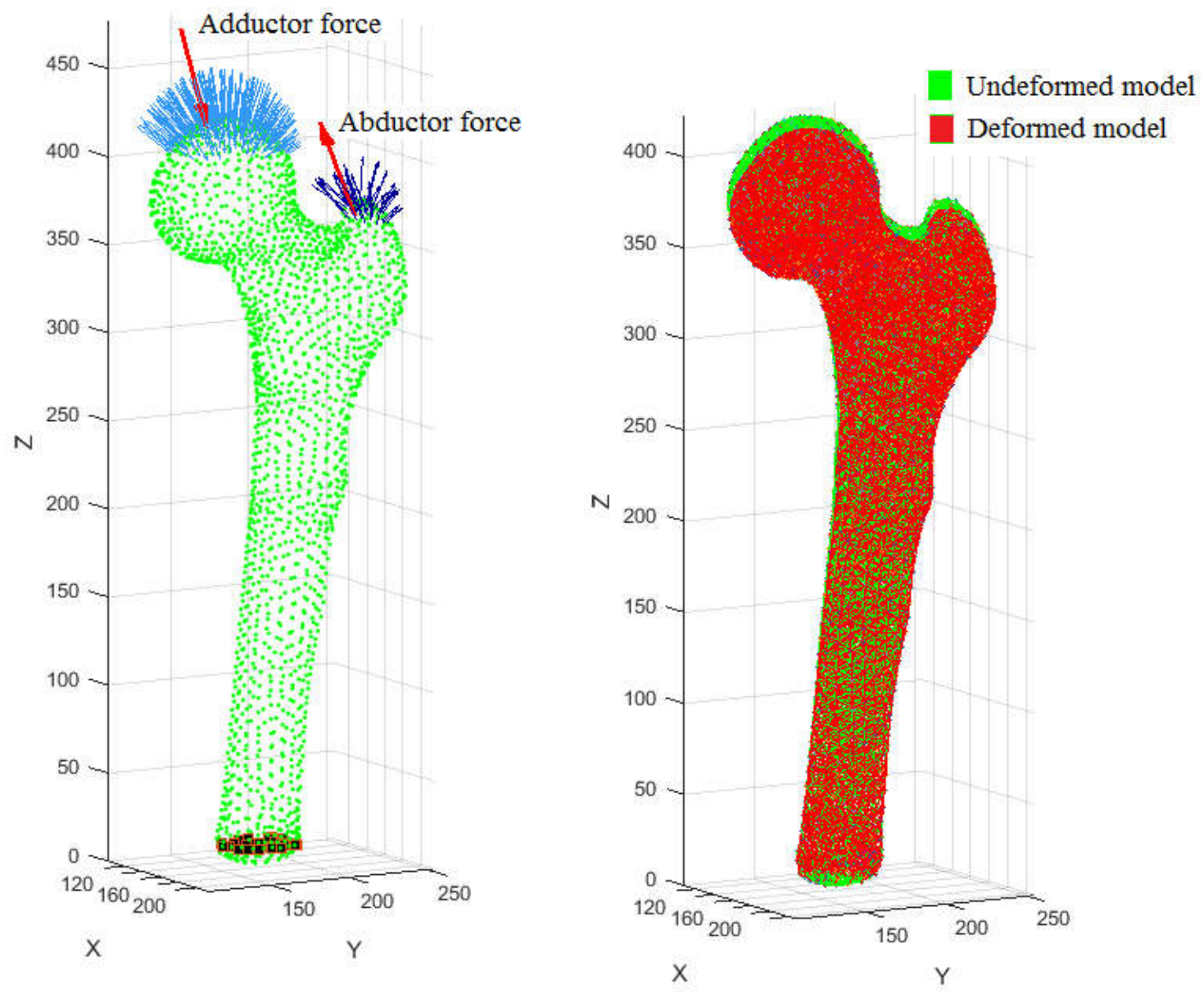

Fig. 7. Boundary conditions applied to the FE model (left) and undeformed/deformed configurations(right).

\section{RESULTS}

A fractured femur CT scanned was reconstructed in 3D solid and analyzed by a standard finite element framework developed by the authors. The ICP algorithm used to align the point clouds data seems to be very effective in this purpose. The inhomogeneous isotropic material properties accessed from CT images were assigned to the FE model. The range of elastic modulus obtained in the procedure of mapping was $1.06-19.46 \mathrm{GPa}$, which fits in the range 
proposed by recent studies. Fig. 7 shows the applied boundary conditions and the deformed configurations obtained after simulation.

Some previous works has been adopted Von Mises equivalent stress as a local yield criterion, as pointed by [22]. Von Mises stress is suitable for materials that present a ductile behavior as metals. On the other hands, in the case of bone the tensile strength is smaller than the compressive strength, indicating that bone be classified as a brittle material, as discussed in [22] and [23]. Thus we adopted here the Drucker-Prager equivalent stress, which takes in account the hydrostatic stress contribution, following the work of [22]. Drucker-Prager yield criterion is described by the following equations:

$$
\begin{aligned}
& F(\sigma)=\alpha J_{1}+\left(J_{2}^{\prime}\right)^{1 / 2} \\
& J_{1}=\sigma_{x}+\sigma_{y}+\sigma_{z} \\
& J^{\prime}{ }_{2}=\frac{1}{2}\left({\sigma^{\prime}}_{x}{ }^{2}+{\sigma^{\prime}}^{\prime}{ }^{2}+{\sigma^{\prime}}_{z}{ }^{2}\right)+\tau_{x y}^{2}+\tau_{y z}^{2}+\tau_{x z}^{2} \\
& \sigma^{\prime}{ }_{i j}=\sigma_{i j}-\delta \sigma_{m} \text { and } \sigma_{m}=\frac{1}{2} \sigma_{i i}
\end{aligned}
$$

where $F(\sigma)$ is a yield function, $\sigma$ is the stress tensor, $\sigma_{x}, \sigma_{y}$ e $\sigma_{z}$ are the normal stress, $\tau_{x y}$, $\tau_{y z}$, and $\tau_{x y}$ are shear stress, the prime (') indicates deviatoric stress. $J_{1}$ is the first stress invariant, $J_{2}$ the second deviatoric stress invariant, and $\alpha$ is a parameter related to the propor-

\begin{tabular}{|c|c|c|c|}
\hline & & $\begin{array}{l}\text { Inhomogeneous } \\
E_{\text {cortical }}=10200 \rho_{\text {ash }}^{2.01}[\mathrm{MPa}] \\
E_{\text {trabecular }}=5307 \rho_{\text {ash }}+469\end{array}$ & $\begin{array}{l}\text { Homogeneous } \\
E_{\text {cortical }}=18100 \mathrm{MPa} \\
E_{\text {trabecular }}=15200 \mathrm{MPa} \\
\end{array}$ \\
\hline \multicolumn{2}{|l|}{ Drucker-Prager eq. stress [MPa] } & 537.85 & 528.28 \\
\hline \multirow[t]{2}{*}{ Principal stress $\quad[\mathrm{MPa}]$} & Max (tensile) & 417.71 & 407.95 \\
\hline & Min (compressive) & -806.32 & -641.97 \\
\hline \multirow[t]{2}{*}{ Principal strains } & Max (tensile) & 0.034099 & 0.022277 \\
\hline & Min (compressive) & -0.073072 & -0.035104 \\
\hline
\end{tabular}
tions of the volumetric and deviatoric strains or the dilative potential of material. Here $\alpha$ is set to 0.07 , as proposed in [24]. Fig. 8 shows the Drucker-Prager equivalent stress evaluated for this structural analysis.

Table 1. Comparison between results obtained by inhomogeneous and homogeneous FE model

In order to compare the effectiveness of the procedure of mapping inhomogeneous isotropic material properties, we perform the same structural analysis considering a material with homogeneous distribution of elastic modulus. It was adopted here two constant values for elastic modulus: $18.1 \mathrm{GPa}$ for cortical bone and $15.2 \mathrm{GPa}$ for trabecular bone, as proposed by [25]. The results are summarized at Table 1. The values for Drucker-Prager equivalent stress and tensile principal stress are similar. Significant differences are observed for compressive values of principal stress and strains, what suggests that inhomogeneous model capture better the mechanical behavior on compression. The contribution of hydrostatic stress in the yield criterion on Drucker-Prager equivalent stress also reveals some remarks. As can be seen in the equations 7 and $8, J_{1}$ becomes negative when hydrostatic stress is presented, what means that the yield criterion $F(\sigma)$ becomes smaller. So as discussed by [22], a larger external load is necessary to cause the yielding of an element. 


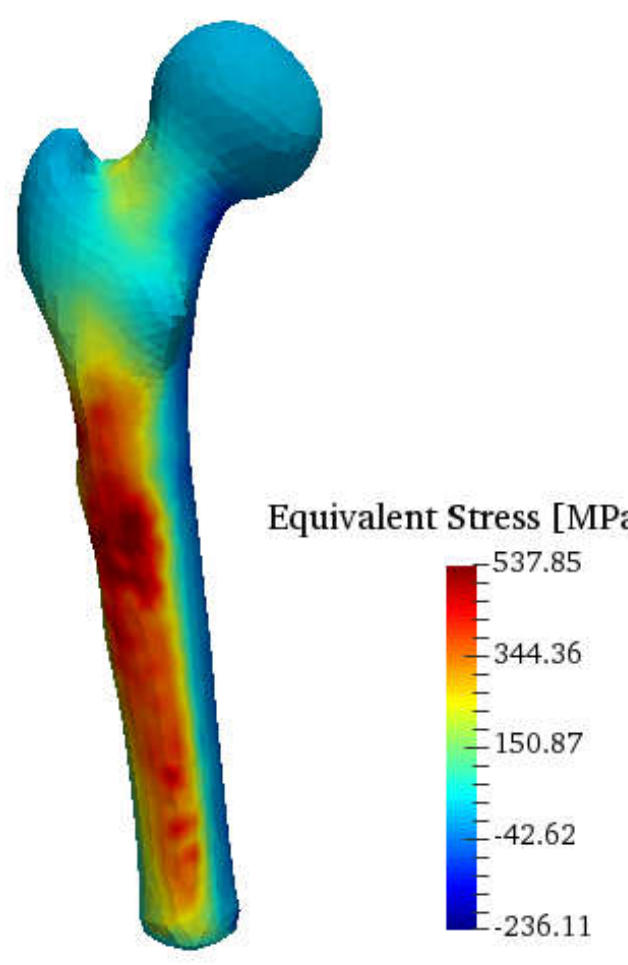

Fig. 8. Drucker-Prager equivalent stress distribution for FE model with inhomogeneous elastic modulus.
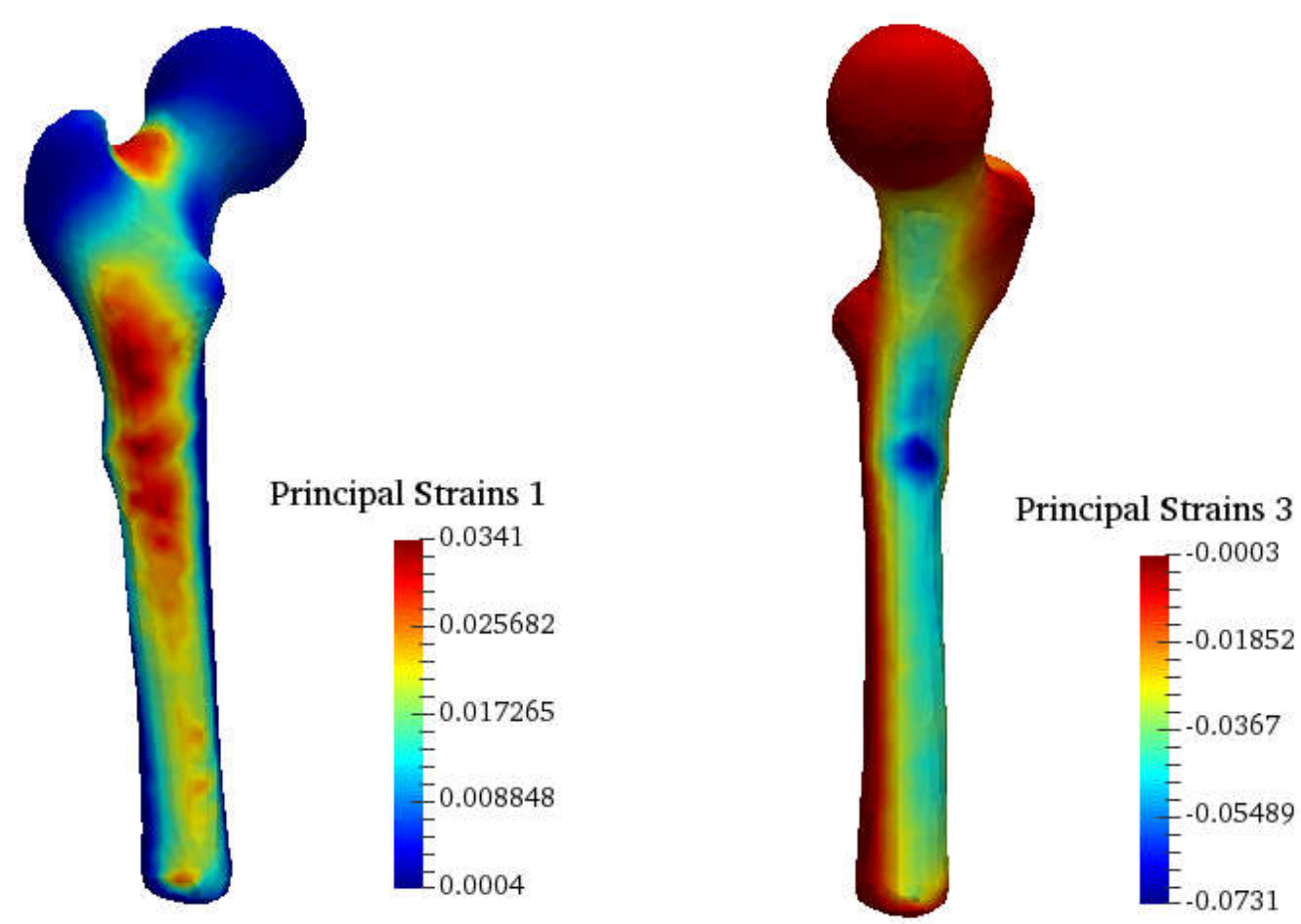

Fig. 9. Maximum (tensile) e minimum (compressive) principal strains (left and right, respectively) in FE model with inhomogeneous elastic modulus. 
The maximum Drucker-Prager equivalent stress obtained in this simulation for inhomogeneous and homogeneous models were rather closer, $537.85 \mathrm{MPa}$ and $528.28 \mathrm{MPa}$, respectively. These values will be used in a future work to access whether the equivalent stress exceed the element yield stress, which allows to evaluate locally the failure sites as shown in [22].

The maximum (tensile) and minimum (compressive) principal strains are shown on Figs. 9 and 10 for inhomogeneous and homogeneous elastic modulus. The results for tensile principal strains were similar but there were significant differences for compressive principal strains. The values for maximum and minimum principal strains are also are listed on Table 1. An important result can be observed in these figures. The regions which present maximum and minimum principal strains (tensile and compressive) are in a region very close to the real fracture, indicating that this model can be used to predict the failure site in a perspective of subject-specific FE models.
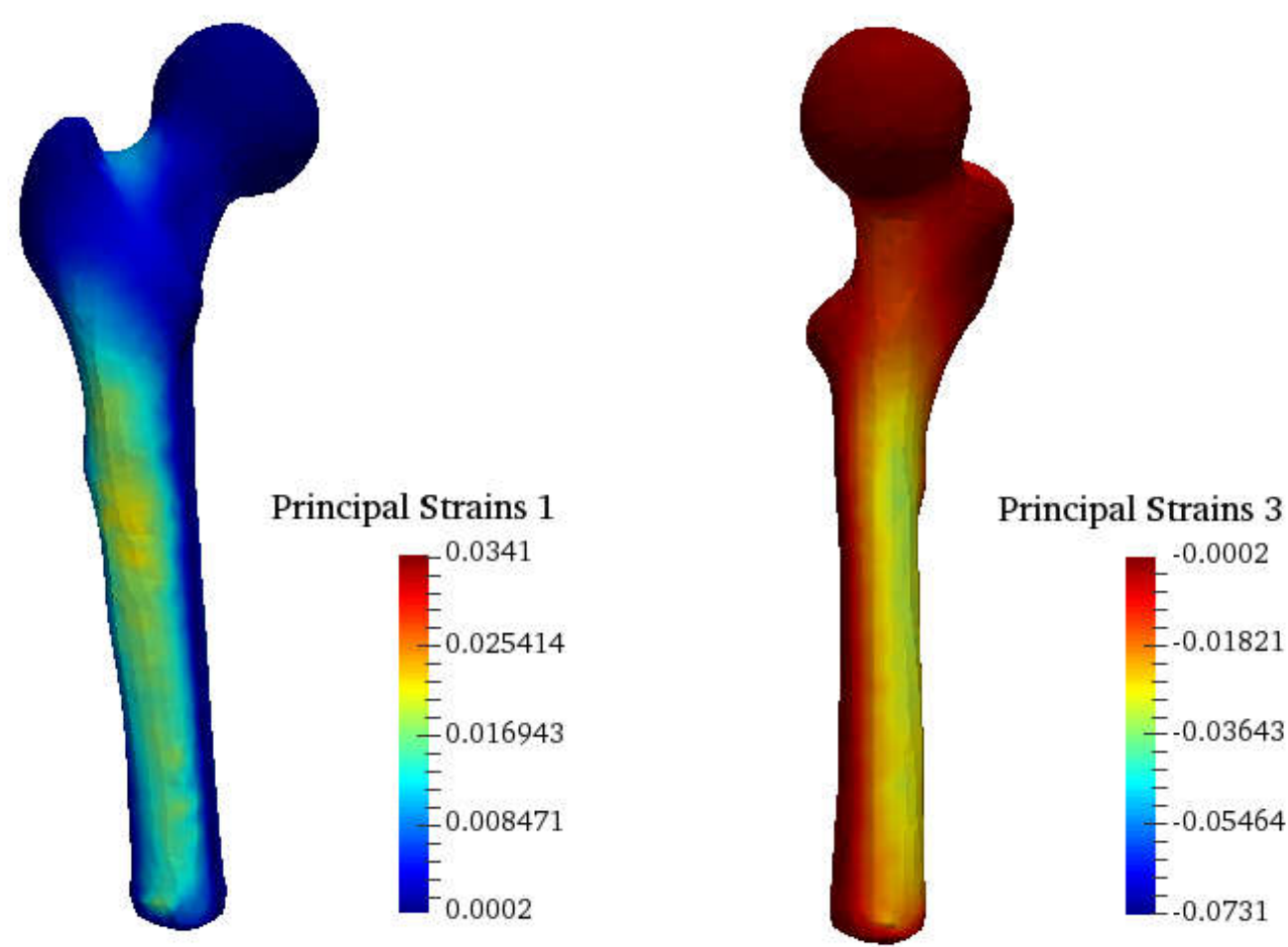

Fig. 10. Maximum (tensile) e minimum (compressive) principal strains (left and right, respectively) in FE model with homogeneous elastic modulus.

\section{DISCUSSION}

Perform a structural analysis in a reconstructed bone as show in this work presents some limitations. First of all, there is rarely specific experimental data for each patient under study to compare with the numerical simulation, which led to adopt values available in the literature. This option may seem rather strange considering that we are interested in a subject-specific model. Other important note is the impossibility to recover the real boundary conditions (muscle and ligaments forces, joint reactions) involved before fracture. So it is very difficult to achieve in the numerical simulation the same location of the real fracture without knowledge and strict control of the boundary conditions. Choose real or almost real boundary conditions constitute a major challenge in this type of numerical simulation. In order to over- 
come this challenge or at least minimize its effects it is convenient analyse different scenarios of loading to cover the entire range of loading cases.

The bone modulus-density relationships available in the literature have been demonstrated good agreement with experimental data. Unfortunately, there are few works evaluating the effectiveness of these relationships when we are dealing with specific cases presenting bone diseases, such as osteoporosis and osteopenia cases.

\section{CONCLUSIONS}

- The access of material properties from CT images offers many possibilities to the biomechanics structural analysis. Reliable subject-specific FE models require information about the inhomogeneities materials properties of each particular case in study. The use of the medical image data, however, requires caution considering that obtaining reliable values of bone ash density (used in the modulus-density relationships) must consider the type of CT equipment, calibration, the type of tissue under study, and ideally should have an experimental support for ensure validation

- The procedure presented here for reconstruction of a fractured bone preserving the CT image information offers a new perspective to analyze the causes of bone fractures since allows recover the material state immediately after the failure. Find real boundary conditions remains a challenge, but the results obtained in this research offers new perspectives to investigate.

\section{REFERENCES}

[1] M. Doblaré, J. M. García, Anisotropic bone remodelling model based on a continuum damage-repair theory. Journal of Biomechanics 35(1): 1-17, 2002.

[2] E. Schileo, E. Dall'ara, F. Taddei, A. Malandrino, T. Schotkamp, M. Baleani, M. Viceconti, An accurate estimation of bone density improves the accuracy of subject-specific finite element models. Journal of Biomechanics 41:2483-2491, 2008.

[3] E. Schileo, F. Taddei, A. Malandrino, L. Cristofolini, M. Viceconti, Subject-specific finite element models can accurately predict strain levels in long bones. Journal of Biomechanics 40, 2982-2989, 2007.

[4] M. Viceconti, M. Davinelli, F. Taddei, A. Cappello, Automatic generation of accurate subject-specific bone finite element models to be used in clinical studies. Journal of Biomechanics 37, 1597-1605, 2004

[5] J. Y. Rho, M. C. Hobatho, R. B. Ashman, Relations of mechanical properties to density and CT numbers in human bone. Medical Engineering and Physics, Vol. 17, No. 5, 347-355, 1995.

[6] D. R. Carter, W. C. Hayes, The compressive behavior of bone as a two-phase porous structure. The Journal of Bone \& Joint Surgery, 59(7):954-62, 1977.

[7] F. Taddei, A. Pancanti, M. Viceconti, An improved method for the automatic mapping of computed tomography numbers onto finite element models, Medical Engineering \& Physics, 26: 61-69, 2004. 
[8] H. M. Kjer, J. Wilm, Evaluation of surface registration algorithms for PET motion correction, Bachelor thesis, Technical University of Denmark, Informatics and Mathematical Modelling, 2010

[9] Y. Chen and G. Medioni. Object modeling by registration of multiple range images. Proceedings. 1991 IEEE International Conference on Robotics and Automation, pages 2724-2729 vol.3, 1991.

[10] P. J. Besl and H. D. McKay. A method for registration of 3-d shapes. IEEE Transactions on Pattern Analysis and Machine Intelligence, 14(2):239-256, 1992.

[11] K. S. Arun, T. S. Huang, and S. D. Blostein. Least-squares fitting of two 3-d point sets. IEEE Transactions on Pattern Analysis and Machine Intelligence, PAMI-9(5):698-700, 1987.

[12] N. Trabelsia, C. Milgrom, Z. Yosibash, Patient-specific FE analyses of metatarsal bones with inhomogeneous isotropic material properties. Journal of Mechanical Behaviour of Medical Materials, 20: 177-189, 2014.

[13] K. H. Kristen, , K. Berger, , C. Berger, , W. Kampla, , W. Anzbock, , S.H. Weitzel, The first metatarsal bone under loading conditions: a finite element analysis. Foot and Ankle Clinics of North America 10 (1), 1-14, 2005.

[14] J. M. Garcia-Aznar, J. Bayod, A. Rosas, R. Larrainzar, R. Garcia-Bogalo, M. Doblare, L.F. Llanos, Load transfer mechanism for different metatarsal geometries: a finite element study. Journal of Biomechanical Engineering 131 (2) 021011-1-021011-7, 2009.

[15] Y. D. Gu, X. J. Ren, J. S. Li, M. J. Lake, Q. Y. Zhang, Y. J. Zeng, Computer simulation of stress distribution in the metatarsals at different inversion landing angles using the finite element method. International Orthopaedics 34 (5), 669-676, 2010.

[16] T. S. Keller, Predicting the compressive mechanical behavior of bone. Journal of Biomechanics 27, 1159-1168, 1994.

[17] Yosibash, Z., Trabelsi, N., Milgrom, C., Reliable simulations of the human proximal femur by high-order finite element analysis validated by experimental observations. Journal of Biomechanics 40 (16), 3688-3699, 2007.

[18] N. Trabelsi, , Z. Yosibash, , C. Milgrom, Validation of subjectspecific automated p-FE analysis of the proximal femur. Journal of Biomechanics 42 (3), 234-241, 2009.

[19] C. Zannoni, R. Mantovani, M. Viceconti, Material properties assignment to finite element models of bone structures: a new method. Medical Engineering \& Physics, 20: 735-740, 1998.

[20] Firoozbakhsh K, Cowin SC. An analytical model of Pauwels' functional adaptation mechanism in bone. Journal of Biomechanical Engineering. Nov; 103(4):246-52, 1981.

[21] J. Nedoma, J. Stehlik, Mathematical and Computational Methods and Algorithms in Biomechanics: Human Skeletal Systems, John Wiley \& Sons, 2011.

[22] M. Bessho, I. Ohnishi, J. Matsuyama, T. Matsumoto, K. Imai K, K. Nakamura, Prediction of strength and strain of the proximal femur by a CT-based finite element method, Journal of Biomechanics, 40(8):1745-53, 2007.

[23] J. Cordey, E. Gautier, Strain gauges used in the mechanical testing of bones Part I: Theoretical and technical aspects, Injury,30 (Suppl 1): A7 - 13, 1999. 
[24] H. B. Kupfer, K. H. Gerstle, Behavior of Concrete Under Biaxial Stresses, Journal of Engineering Mechanical Division, ASCE 99, 853 - 866, 1973.

[25] H. H. Bayraktar, E. F. Morgan, G. L. Niebur, G. E. Morris, E. W. Wong, T. M. Keaveny, Comparison of the elastic and yield properties of human femoral trabecular and cortical bone tissue, Journal of Biomechanics, Volume 37, Issue 1, 27 - 35, 2004. 